\title{
Bienestar Subjetivo y Tipo de Vinculación en una entidad pública del orden municipal: Un estudio Predictivo
}

\author{
Lina María Espinal Ramírez ${ }^{1}$, Juliana Ximena Trujillo² \& Alejandro Sanín Posada
}

Recibido: 18/06/2018 Aceptado: 25/09/2018

DOI: $10.21772 /$ ripo.v37n1a02

\begin{abstract}
Resumen
El objetivo del presente estudio fue explorar qué tanto el tipo de vinculación laboral predice el nivel de bienestar subjetivo en el trabajo. Para ello se trabajó con una muestra de 333 trabajadores de una organización pública del orden municipal. Aplicándose la escala F7, de Felicidad laboral a todos ellos. Posteriormente, se hizo un análisis de varianza múltiple para identificar qué tanto la variable dependiente difería en función de la pertenencia a diversos tipos de contrato laboral tenidos en cuenta como variable categórica. Los resultados no permitieron confirmar la hipótesis de estudio, se encontró ausencia de diferencias estadísticas significativas cuando se compararon los resultados por tipo de vinculación. Esto indica que las realidades asociadas a uno u otro tipo de vinculación no parecen estar afectando los niveles de bienestar de las personas.
\end{abstract}

Palabras clave: bienestar subjetivo, tipos de vinculación, entidad pública.

\section{Subjective Wellbeing and Type of Employment contract in a municipal entity: A Predictive study}

\begin{abstract}
This study explores whether the type of employment contract predicts the subjective overall well-being in the workplace. For this, we applied the F7 Workplace Happiness Survey to a sample of 333 employees in a municipal entity. MANOVA was performed in order to identify whether the dependent variable varies with the type of employment contract, as a categorical variable. The resulting absence of statistically significant differences between the employment contract fails to confirm the study hypotheses. This suggests that the conditions imposed by each type of employment contract do not seem to make a difference in the overall well-being of the employee.
\end{abstract}

Keywords: subjective well-being, type of employment contract, public entity

1 Psicóloga - Universidad de San Buenaventura. Magister en Desarrollo Humano Organizacional- Eafit. Especialista en Gerencia del Desarrollo HumanoEafit. Especialista en Salud y Seguridad en el Trabajo - UdeA. Gerente de Gestión Humana. E-mail: espinalramirezlina@gmail.com 2 Abogada. Especialista en Gerencia del Desarrollo Humano- Eafit. Magister en Desarrollo Humano Organizacional- Eafit. Alcaldía de Envigado. Abo-
gada contratista dirección de compras secretaria general. E-mail: julianatrujillo86@hotmail.com

$3 \mathrm{PhD}$, en Psicología del Trabajo y las Organizaciones. Centro de Investigación en Comportamiento Organizacional Cincel. Director de Proyectos. Medellín - Colombia. Email: asaninp@cincel.com.co

Cómo citar este artículo: Espinal Ramírez, L. M.; Trujillo, J. X. \& Sanín Posada, A. (2018). Bienestar subjetivo y tipo de vinculación en una entidad pública del orden municipal: un estudio predictivo. Revista Interamericana de Psicología Ocupacional, 37(1), 17-25. DOI: 10.21772/ripo.v37n1a02 


\section{Introducción}

La psicología positiva es una perspectiva teórica de la psicología que ha puesto especial atención en condiciones de salud, el bienestar de las personas y en describir las formas y causas que explican cómo los seres humanos se adaptan adecuadamente a los diferentes entornos (Seligman, 2016). La felicidad hace parte de esos conceptos que aborda con rigurosidad dicha disciplina. Sin embargo, en el lenguaje científico y académico se utiliza el concepto de Bienestar Subjetivo en lugar del de felicidad por considerarse este último más de uso común (Diener, 2000).

En esa línea se encuentra Seligman (2016), uno de los autores más reconocidos en el abordaje científico y académico de los fenómenos psicológicos positivos, quien en sus primeros trabajos y aportes científicos se refería al constructo como felicidad, ha conservado la definición, pero lo ha nombrado bienestar subjetivo pretendiendo dotarlo de mayor rigurosidad científica (Sanín, 2017).

En el presente estudio ambos conceptos, felicidad y bienestar subjetivo, serán abordados como sinónimos, siguiendo la línea de Diener (2000) y Seligman (2016). Se entenderá el bienestar subjetivo, como un estado afectivo positivo caracterizado por la presencia de emociones positivas, gratificación y sentido (Seligman, 2011). El concepto es susceptible de ser adaptado al contexto laboral, pues es un entorno en el que también puede haber disfrute, sentido y gratificación (Sanín, 2017).

Las definiciones más recientes del constructo consideran dos dimensiones del bienestar, a saber, el sentido de lo que se hace y la gratificación (Seligman, 2011). La primera de éstas se refiere a la comprensión del propósito de lo que se hace. La segunda a la posibilidad de sentir que eso resulta enriquecedor, formativo y da identidad, por cuanto implica se han utilizado las fortalezas características del ser humano (Seligman, 2011). El bienestar subjetivo laboral requiere la existencia de emociones positivas, gratificaciones y sentido del trabajo. Su evaluación implicaría examinar en cuál medida la persona considera que, en la entidad a la que está vinculado o través del desarrollo de su trabajo, puede sentirse bien (bienestar), desarrollar actividades que le exigen la aplicación de sus fortalezas (gratificación) y si encuentra sentido y propósito a su trabajo.

El estudio y la investigación científica del bienestar subjetivo pueden resultar de interés para diversos investigadores de múltiples campos del sabery disciplinas. En este caso, se pretendehacerlo en el contexto del trabajo. Dicha preferencia radica en las múltiples investigaciones que sugieren que el bienestar tiene efectos importantes en la productividad individual y laboral (Seligman, 2011). Además, representa un propósito de vida que no requiere de justificación alguna, pues se trata de una búsqueda que caracteriza a los seres humanos como especie (Seligman, 2011).

La revisión bibliográfica permitió identificar que en las investigaciones sobre bienestar subjetivo, realizadas en los últimos años, se tiende a relacionar el constructo con aspectos como la motivación y las estrategias de afrontamiento (Sanjuán \& Ávil, 2016), la satisfacción vital (Denegri, Castaño, Schenettler \& Vivallo, 2016) (Laca, 2015), la calidad de vida, el bienestar físico y psico-social (Flórez, 2015), las emociones (González-Villalobos \& Marrero, 2017), la personalidad (Marcial, Cabrera \& Rosario, 2015), y sus niveles en poblaciones como directivos (Reyes, López \& Reyes, 2016), personas mayores (Flecha-García, 2015) (Fernández, Clúa, Dueñas, Ramírez \& Prieto, 2000) (Domínguez, Mandujano, López, Domínguez, González, Sotomayor \& Fraijo, 2011) entre otros. Sin embargo, no se encontraron hallazgos asociados con el tipo de vinculación, en el sector público en Colombia. ${ }^{4}$

\footnotetext{
4 Búsqueda realizada en julio de 2017 en EBSCO Discovery Service y Publication Finder Interface utilizando las palabras "Bienestar Subjetivo", "Tipo de vinculación", "Felicidad en el trabajo"," Subjective well-being".
} 
Aunque la vinculación laboral ha sido un tema abordado principalmente por el derecho y las ciencias económicas, en los últimos años, ha comenzado a ser revisado desde una perspectiva social y psicológica, pues se reconocen sus posibles efectos sobre las personas, la sociedad y las organizaciones (Sanín, 2017). El tipo de vinculación laboral puede afectar el contrato psicológico (Alcover, Martínez-Inigo \& Chambel, 2012) y éste, a su vez, influenciar el rendimiento, la motivación, el ausentismo, los comportamientos extra rol, el compromiso, la satisfacción y el bienestar psicológico (Rousseau, 1995).

El contrato psicológico se refiere a las relaciones basadas en el intercambio que surge entre empleados y empleadores (Chambel \& Alcover, 2011). Cada parte tiene la expectativa de recibir algo a cambio más allá de lo establecido en el contrato legal. Es decir, se forman creencias entre las partes sobre lo que obtendrán de dicha relación (Conway \& Briner, 2005).

En el caso del trabajo en las instituciones públicas, la vinculación con el estado suele ser deficiente (Aguilera, 2011), generando con ello particularidades en la forma como se configura el contrato psicológico. Buen ejemplo de ello, es que en el ámbito público los trabajadores o funcionarios pueden verse afectados frente a cualquier pretensión arbitraria del poder político (Aguilera, 2011). Mientras para una terminación del vínculo laboral, en el ámbito privado, hay margen para el acuerdo entre las partes o la decisión unilateral que opera bajo el principio de autonomía de la voluntad. En lo público, por el contrario, se debe actuar con sometimiento pleno a la ley y al derecho (Aguilera, 2011).

Esta diferencia nos muestra oportunidades de desarrollo de conocimiento científico. Se trata de la comprensión de cómo las particularidades del trabajo en el sector público y sus exclusivos y diferentes tipos de vinculación crean o promueven condiciones sociales y legales que puedan afectar el bienestar subjetivo del trabajador, dados los posibles efectos que sobre el contrato psicológico pueden tener. De esta manera, si se sabe que el contrato psicológico tiene efectos sobre el bienestar de las personas, y que los tipos de vinculación en lo público pueden hacer que ese contrato sea diferente, es lícito preguntarse si el bienestar subjetivo varía o no dependiendo del tipo de vinculación de las personas en una entidad pública del orden municipal. Este conocimiento servirá de sustento para la formulación de políticas públicas que propendan por la búsqueda del bienestar en contextos organizaciones, en especial el sector público.

En la presente investigación se abordaron diferentes formas de vinculación en el sector público en Colombia, que pueden encontrarse en diversas entidades independientes del orden territorial. Dichos tipos de vinculación son abordados por la legislación Colombiana en especial por la Constitución política colombiana en su artículo 122, Ley 909 de 2004, decreto 3135 de 1968, decreto 1333 de 1986 y Ley 80 de 1993. Según esta normatividad, en Colombia existen dos tipos de vinculación laboral con el Estado: la legal y reglamentaria, de la cual hacen parte los denominados empleados de carrera administrativa. Son empleos que la administración pública oferta mediante concurso de méritos, sin que en éste se tenga en cuenta la discrecionalidad del empleador para proveerlos. Su nombramiento se hace después de pasar por todo un proceso de mérito que se desarrolla en su gran mayoría por la comisión nacional del servicio civil, luego de que las entidades estatales reporta los cargos que se encuentran disponibles para ser ofertados; provisionalidad son empleos que en su naturaleza son de carrera administrativa pero que a la fecha no están siendo ofertados por concurso de méritos; en este empleo se hace un nombramiento en provisionalidad y la persona que se nombra se hace a discrecionalidad de la entidad siempre que cumpla los requisitos para el cargo. Este tipo de vinculación tiene las mismas características de un empleo de carrera administrativa con la diferencia 
que cuando es ofertado por concurso de méritos y se suple la necesidad, el funcionario es retirado de la provisionalidad. Y libre nombramiento y remoción son empleos provistos mediante acto administrativo de nombramiento o nombramiento ordinario, y acta de posesión en el empleo; el nombramiento se realiza previo al cumplimiento de los requisitos exigidos para el desempeño del empleo; como su nombre lo indica son empleos en relación con los cuales la autoridad competente para su nombramiento y remoción puede tomar las medidas pertinentes en forma discrecional.

La segunda forma o manera de vincularse con las entidades estatales es la denominada por la norma como la laboral contractual. De ella hacen parte los trabajadores oficiales, quienes a diferencia de los empleados públicos son vinculados mediante contrato de trabajo y tanto su permanencia como desvinculación deben ceñirse a las normas que rigen la materia y las disposiciones del Código Sustantivo de Trabajo.

También existe otra modalidad de vinculación en las entidades estatales, los contratos de prestación de servicios. En este caso no se da una relación laboral directa con la entidad. Estos contratos son celebrados para desarrollar actividades relacionadas con la administración o funcionamiento de la entidad en los casos en los que dichas actividades no puedan ser desarrolladas con personal de planta (artículo 32 de la Ley 80 de 1993).

Por lo anterior, y en relación con hallazgos de investigadores anteriores que muestran que el Tipo de Vinculación afecta la satisfacción laboral (Santos, Guillén \& Montalbán, 2012) y el bienestar de las personas en el trabajo (Alcover, et al., 2012), se hipotetiza que el tipo de vinculación laboral podría permitir predecir los niveles de bienestar subjetivo en el trabajo de las personas, en términos de sus emociones positiva, su gratificación y el sentido que le encuentran al trabajo. Las razones para pensar esto son que las distintas modalidades de vinculación hacen que las personas estén más o menos seguras en sus puestos de trabajo en tanto, algunos son fácil, en términos administrativos, de remover de sus cargos, y la seguridad de los mismos depende en gran medida de los periodos gubernamentales que caracterizan el sector público en Colombia. Suponemos entonces, que estas particularidades de las entidades públicas pueden llegar a interferir el sentirse bien en el trabajo (bienestar), la posibilidad de desarrollar actividades que exijan la aplicación de fortalezas (gratificación) y el encontrarle sentido y propósito a lo que se hace.

Es apremiante entonces preguntarse si las condiciones asociadas al tipo de vinculación que se tiene con una entidad pueden afectar el bienestar subjetivo, entendido como un fenómeno más general que involucra las emociones positivas, la gratificación y el sentido en el trabajo.

Por lo que, encontrar la respuesta a la pregunta ¿Es el tipo de vinculación un predictor de los niveles de bienestar subjetivo de los trabajadores en una entidad pública del orden municipal? se vuelve un objetivo pertinente no solo para las investigadoras, sino porque la misma permite aportar al conocimiento del constructo y sus determinantes en diferentes contextos laborales, y cómo variables positivas, como el bienestar subjetivo, cambian o no dependiendo del tipo de vinculación que se tenga, en contextos no tan estudiados, como el sector público en Colombia. A su vez, los hallazgos encontrados pueden servir de sustento para la formulación de políticas públicas que propendan por la búsqueda del bienestar en contextos organizaciones, en especial el sector público.

\section{Método}

\section{Procedimiento y participantes}

La recolección de los datos se hizo vía online (100\%). Se pidió siempre el consentimiento informado de los participantes, cumpliendo 
las leyes que regulan la investigación con seres humanos en Colombia (Ministerio de Salud, 1993). Los participantes destinaron aproximadamente 10 minutos en el diligenciamiento de la encuesta que fue publicada en la plataforma docs.google.com.

Los casos corresponden a personas vinculadas a una entidad pública del orden municipal, con la cual se pudo recolectar 333 casos válidos. Aunque participaron más personas sus datos no fueron incluidos, pues no desearon finalizar la encuesta.

La muestra se caracterizó en las siguientes proporciones: Edad (31\% de 51 a 60 años, 28\% de 41 a 50 años, $27 \%$ de 31 a 40 años, 11\% de 20 a 30 años, 3\% de 61 años en adelante); Sexo (54\% mujeres); Estado Civil (48\% Casados(a), $32 \%$ solteros(a), 10\% divorciados(a), 10\% unión libre, $1 \%$ viudos(a)); Tipo de vinculación (42\% provisionalidad, 33\% carrera administrativa, 14\% contratistas, $10 \%$ libre nombramiento y remoción, $1 \%$ trabajador oficial). En contraprestación la entidad recibió un informe particular por permitir la participación de sus colaboradores. Esto se hizo solo cuando la muestra resultó representativa.

\section{Instrumento}

El bienestar Subjetivo, fue evaluado con el instrumento F- SIETE desarrollado por CINCEL (2016), ya validado en población adulta trabajadora colombiana $\left(\alpha=.88 ; \chi^{2}=77,408\right.$, g1 (45) $\mathrm{p}=.000$; CFI y TLI $>$.95; RMSEA $=0.006$, $\mathrm{p}=.267)$. Éste examina el nivel de felicidad en el trabajo de las personas en términos de la vivencia de emociones positivas, obtención de gratificación por aplicación de las fortalezas y experimentación de sentido. Cuenta con 10 ítems que se responde eligiendo una de cuatro opciones posibles que describen niveles de disfrute, gratificación o sentido de las personas (v.g. "en mi trabajo suelo experimentar" (A) alegría la mayor parte del tiempo, (B) alegría y algo de tristeza, (C) tristeza y algo de alegría, (D) tristeza la mayor parte del tiempo).

\section{Análisis de datos}

Se realizaron comparaciones entre grupos usando el Game Howell, aunque los datos fueron no normales se utilizó esta prueba, previa verificación de la homogeneidad de varianzas, por su robustez. El análisis se hizo con el paquete estadístico SPSS Versión 23.

\section{Resultados}

En la Tabla 1 se reporta el Alpha de cada medida, las medias, desviaciones típicas y correlaciones entres las variables del estudio.

Con este banco de datos se aplicó la prueba de normalidad Kolmogorov-Smirnov. Se encontró que los datos eran No normales para Estado Afectivo Positivo $(Z=3.645, p=$ $.000)$, Gratificación en el Trabajo $(Z=4.537, \mathrm{p}=$ $.000)$, Sentido en el Trabajo $(Z=6.683, p=.000)$ y Felicidad en el Trabajo $(Z=3.015, p=.000)$. Se procedió a realizar la comparación entre grupos con Games Howeel verificando primero la homogeneidad de las varianzas. En ningún caso se pudo asumir que la hubiera: Estado afectivo positivo $(\mathrm{p}=.037)$, Gratificación ( $\mathrm{p}$ $=.046)$, Sentido $(\mathrm{p}=.003)$, Felicidad $(\mathrm{p}=.007)$. Por su parte a prueba de contraste reveló que no había diferencias significativas entre los grupos en estado afectivo positivo $(\mathrm{F}=.390, \mathrm{p}=.816)$, gratificación en el trabajo $(\mathrm{F}=1.684, \mathrm{p}=.153)$, sentido en el trabajo $(\mathrm{F}=1.338, \mathrm{p}=.255)$, felicidad en el trabajo $(\mathrm{F}=1.009, \mathrm{p}=.403)$. 
Tabla 1. Fiabilidad, $N$, medias, desviaciones típicas, y correlaciones entre las variables del estudio $(n=333)$

\begin{tabular}{lccccccc} 
& $\alpha$ & $\mathrm{N}$ & Media & DT. & 1 & 2 & 3 \\
\hline 1. Estado Afectivo Positivo en el trabajo & .802 & 333 & 3.5270 & .43650 & - & & \\
2. Gratificación en el trabajo & .783 & 333 & 3.6306 & .46501 & $.390^{* *}$ & - & \\
3. Sentido en el trabajo & .781 & 333 & 3.7888 & .39715 & $.474^{* *}$ & $.513^{* *}$ & - \\
4. Felicidad en el trabajo & .869 & 333 & 3.6488 & .36087 & $.803^{* *}$ & $.795^{* *}$ & $.733^{* *}$ \\
\hline
\end{tabular}

**La correlación es significativa al nivel 0.01 (bilateral).

\section{Discusión}

La presente investigación exploró qué tanto el tipo de vinculación laboral predice el nivel de bienestar subjetivo en el trabajo en los empleados de una entidad pública del orden Municipal.

Los efectos del tipo de vinculación sobre variables psicosociales han sido poco estudiados y los pocos estudios que se encuentran son en organizaciones del sector privado, no del público.

No obstante, los resultados no permitieron confirmar la hipótesis de estudio. Se encontró ausencia de diferencias estadísticas significativas cuando se compararon los resultados por tipo de vinculación. Esto indica que las realidades asociadas a uno $\mathrm{u}$ otro tipo de vinculación no parecen estar afectando los niveles de bienestar de las personas.

Quiere decir que el tipo de vinculación no predice los niveles de bienestar subjetivo de los empleados de la entidad pública estudiada. En este sentido, la gratificación, las emociones positivas y el sentido en el trabajo no parecen depender del tipo de vinculación que se tenga con la organización. Esto es paradójico pues las personas con las cuales se realizó la investigación tenían diferentes tipos de vinculación y se ha encontrado que eso afecta la satisfacción laboral (Santos, et al., 2012), y el contrato psicológico (Alcover, et al., 2012), por lo que intuitivamente se esperaba que el tipo de vinculación afectara significativamente los niveles de bienestar subjetivo.
Se pensaba a su vez que el bienestar subjetivo variaría en función del tipo de vinculación como lo hace en función de algunas variables como la motivación y las estrategias de afrontamiento (Sanjuán \& Ávil, 2016), la satisfacción vital (Denegri, et al., 2016), la calidad de vida, el bienestar físico y psico-social (Flórez, 2015), las emociones (González-Villalobos \& Marrero, 2017), y que esto sería incluso más marcado por tratarse de una entidad pública municipal, las cuales suelen caracterizarse por presentar mayor nivel de vinculación por contratos de prestación de servicios y rotación derivado de cambios de administración.

El hallazgo puede explicarse debido a que las personas suelen creer que ciertas condiciones de vida, como el matrimonio, los hijos, el trabajo y la riqueza los harán felices, sin embargo, estos acontecimientos no son más que una falsa promesa, porque en realidad son las reacciones a ellos las que rigen las repercusiones en el bienestar (MacAdams, Josselson \& Llieblich, 2001). Lo que explicaría que condiciones como el tipo de vinculación laboral pierda su capacidad para influenciar significativamente el bienestar subjetivo de las personas, y más bien aspectos, ya demostrados por los investigadores, como que las personas puedan comportarse en su trabajo de acuerdo con su personalidad, intereses y valores hagan que se sientan más implicadas y satisfechas (Higgings, 2005).

A su vez, el trabajo es una esfera de la vida a la que es susceptible adaptarse hedonistamente 
(Lyubomirsky, 2014), por lo cual, a pesar de haber conseguido el trabajo deseado y bajo la vinculación deseada, las personas terminan acostumbrándose a la novedad y a las condiciones del nuevo empleo. Esto sustentaría otra posible explicación; la felicidad de las personas puede alimentarse de las emociones positivas, la gratificación y el sentido proveniente de otras facetas de las personas (Lyubomirsky, 2014). En consecuencia, el tipo de contrato pierde su capacidad de afectar el bienestar de las personas. Como se observó, la mayoría de los trabajadores de la entidad pública se sitúa dentro de un nivel óptimo de bienestar, esto sugiere que valoran otros aspectos de su vida además del laboral.

La razón de los resultados de esta investigación puede deberse a que, a diferencia de otras variables psicosociales, el bienestar subjetivo o la felicidad son aspectos estructurales de los sujetos (Higgings, 2005), por tanto, su alteración es más difícil y puede resistir incluso las contrariedades derivadas de tipos de vinculación precarios (Sanín, 2017). La causa, es lo que Lyubomirsky (2014) logra resumir en una frase: "la felicidad no proviene de afuera de nosotros; habita en nuestro interior" (p.157).

\section{Conclusiones y limitaciones}

Los resultados del análisis de datos permiten concluir que las personas se adaptan a las condiciones que enfrentan. Si éstas no van en contra del disfrute, la gratificación y el sentido no parece que representen mayor problema, así las cosas, una persona contratada mediante prestación de servicios puede disfrutar y encontrarle tanto sentido a su trabajo como uno vinculado en carrera administrativa, provisionalidad o libre nombramiento y remoción.

Así mismo, se encontró que el tipo de vinculación no incide sobre el bienestar subjetivo de las personas que laboran en una entidad pública del orden municipal. Lo cual puede deberse a que la percepción de bienestar o felicidad hace parte de un conjunto de situaciones personales en donde prima la sumatoria de todos los aspectos de la vida y no sólo su situación o condición laboral lo que se identificó en las puntuaciones obtenidas en la investigación.

La mayoría de personas que participaron en el estudio obtuvieron puntuaciones cercanas al tope superior de la escala, lo que indica niveles de bienestar subjetivo alto.

También se debe tener presente las limitaciones propias de la técnica estadística empleada. Harán falta muestras más amplias y diversas para poder descartar plenamente que no existe relación entre el tipo de vinculación y el nivel de bienestar subjetivo de las personas que laboran en las entidades del sector público.

Otra limitación de la investigación puede radicar en que la aplicación del instrumento se realizó con autorización de la entidad y la misma fue enviada por el departamento de Gestión Humana, lo que podría haber afectado el resultado; ya que a pesar de ser anónima las personas pudieron haber reportado estar mejor de lo que en realidad estaban por temor a mostrarse insatisfechos ante la entidad.

Se recomienda reaplicar el estudio incluyendo otras categorías que permitan verificar qué variables sociodemográficas pueden incidir en el bienestar de los trabajadores.

Este estudio aporta al conocimiento del constructo en tanto se logró concluir que el tipo de vinculación en una entidad pública del orden municipal no es predictor del bienestar subjetivo de las personas y que son más bien otros factores como condiciones asociadas a la pertenencia de variables sociodemográficas o características personales los que pueden afectar la percepción de la gratificación, las emociones positivas y el sentido en el trabajo de las personas (Lyubomirsky, 2014). 
Gracias a este estudio se pudo evidenciar que el tipo de vinculación no es un predictor del bienestar subjetivo sino es analizado con otras variables que afecten directamente la percepción de vida del ser. Es decir, aspectos como condiciones sociodemográficas, psicológicas, condiciones de sexo, edad, familiares y otros aspectos que afecten directamente, sus emociones positivas, gratificación y el sentido que le dan su vida y a el trabajo.

\section{Referencias}

Alcover, C. M., Martinez-Inigo, D., \& Chambel, M. J. (2012). Perceptions of employment relations and permanence in the organization: Mediating effects of affective commitment in relations of psychological contract and intention to quit. Psychological Reports, 110(3), 839-853. Doi: 10.2466 / 01.07.21.PR0.110.3.839- 853

Aguilera, R. (2011). La extinción del contrato de trabajo del personal al servicio de las Administraciones Públicas. Revista del Ministerio de Trabajo y Asuntos Sociales, 93, 317-342.

Chambel, M. \& Alcover, C. (2011). The psychological contract of call-centre workers: Employment conditions, satisfaction, and civil virtue behaviours. Economic and Industrial Democracy. 32 (1), 115-134.

Cincel (2016). F-Siete Ficha técnica. Documento Inédito.

Constitución Política de Colombia, segunda edición corregida, publicada en la gaceta constitucional Número 116 de 20 julio de 1991.

Conway N. \& Briner R. (2005). Understanding Psychological Contracts at Work. A Critical Evaluation of Theory and Research. Oxford: Oxford University Press.

Decreto 1333. Cámara de comercio, Bogotá, Colombia, 25 de abril de 1986.

Decreto 3135. Bogotá, Colombia, 26 de diciembre de 1968.
Denegri, M., Castaño, A., Schenettler, B. \& Vivallo, O. (2016). Bienestar Subjetivo y Patrones de alimentación en adultos chilenos: un estudio cualitativo. Revista Mexicana de Trastornos alimenticios, 7 (2). 105-115.

Diener, E. (2000). Subjective well-being: The science of happiness and a proposal for a national index. American Psychologist, 55(1), 34-43. Doi: 10.1037/0003-066X.55.1.34

Domínguez, M., Mandujano, M., López, G., Domínguez, R., González, M., Sotomayor, M. \& Fraijo, B. (2011). Escala de Bienestar Subjetivo en Cuidadores Familiares de Adultos Mayores (EBEMS/CFAM). Revista de Psicología, 29 (2), 265-287.

Fernández, N., Clúa, A., Dueñas, R., Ramírez, M. \& Prieto, V. (2000). Estilos de vida, bienestar subjetivo y salud de los ancianos. Revista Cubana Medicina General Integral,16(1), 6-12.

Flecha-García, A. (2015). Bienestar psicológico subjetivo y personas mayores residentes. Pedagogía Social. Revista Interuniversitaria, 25, 319-341. Doi:10.7179/PSRI_2015.25.14

González-Villalobos, J. \& Marrero, R. (2017). Determinantes sociodemográficos y personales del bienestar subjetivo y psicológico en población mexicana. Suma Psicológica, 24 (1), 59-66.

Higgins, E. (2005). Value from regulatory fit. Current directions in psychological science, 14(4), 209-213.

Laca, F. (2015). El largo camino hacia el estudio científico de la satisfacción con la vida y el bienestar subjetivo. Pensamiento Americano, $8(15), 27-40$.

Ley 80 de 1993, diario oficial número 41094 Republica de Colombia, Bogotá, Colombia, 28 de octubre de 1993.

Ley 909 de 2004, diario oficial número 45680 República de Colombia, Bogotá, Colombia, 23 de septiembre de 2004. 
Lyubomirsky, S. (2014). Los mitos de la felicidad. Barcelona, España, Editorial Urano.

McAdams, D., Josselson, R., \& Lieblich, A. (2001). Turns in the road: Narrative studies of lives in transition. American Psychological Association.

Marcial, E., Cabrera, D. \& Rosario, J. (2015). Motivos, personalidad y bienestar subjetivo en el voluntariado. Anales de psicología, 31(3), 791801.

Resolución 8430. Ministerio de Salud, Republica de Colombia, Bogotá, Colombia, 04 de octubre de 1993.

Reyes, L., López, R. \& Reyes, A. (2016). Bienestar subjetivo en directivos de la Empresa de Transporte de Oriente. Revista Encuentros.14(2), 89-103.

Rousseau, D. (1995). Psychological Contracts in Organizations: Understanding written and unwritten agreements. London: Sage
Sanín, S. (2017). Felicidad y optimismo en el trabajo. Hallazgos de investigaciones cientificas [Tesis Doctoral], 12-20. http://www.want.uji.es/ tesis-alejandro-sanin/

Sanjuán, P. \& Ávil, M (2016). Afrontamiento $\mathrm{y}$ motivación como predictores del bienestar subjetivo y psicológico. Revista de Psicopatología y Psicología Clínica, 21 (1), 1-10.

Santos, F., Guillén, C. \& Montalbán, M. (2012). Contrato de trabajo, compromiso y satisfacción: moderación de la empleabilidad. Administração de Empresas, 52(3), 345-359.

Seligman, M. (2011). La Autentica Felicidad. Barcelona, España, Ediciones ZETA.

Seligman, M. (2016). Florecer: La nueva psicología positiva y la búsqueda del bienestar. Editorial Océano. 